\title{
ACTUALIZACIÓN EN ACROMEGALIA
}

William Rojas G., MD.*

\section{Introducción}

La acromegalia en una enfermedad causada por la producción anormal de hormona del crecimiento en momentos en los que los huesos ya han tenido su cierre epifisiario, es decir, se presenta en la población adulta de ambos sexos. Cuando esta anormalidad se evidencia en población pediátrica o que no ha terminado su crecimiento, se denomina gigantismo.

Pese a que la acromegalia es una enfermedad de fácil identificación en sus etapas tardías por las alteraciones fenotípicas que produce, este mismo hecho hace que al momento del diagnóstico las complicaciones como la cardiopatía hipertrófica, la artropatía y las lesiones visuales y de la estructura ósea ya estén establecidas y, excepto por el beneficio metabólico que brindamos al tratar el tumor hipofisiario, es poco lo que se puede hacer por las otras afecciones que en la mayoría de los casos son irreversibles. Sin embargo, los avances en las técnicas de diagnóstico imagenológico y la medición de hormonas permiten hacer un diagnóstico precoz en la actualidad, incluso cuando aún no son muy evidentes los cambios fenotípicos de la enfermedad. Esto trae como consecuencia un beneficio para el paciente por la prevención de las complicaciones.

Por lo tanto, el objetivo de este artículo es, además de hacer una revisión sobre los nuevos conceptos fisiopatológicos y de tratamiento de la enfermedad, llamar la atención sobre los síntomas precoces que le puedan hacer sospechar al médico la presencia de esta anomalía endocrina.

\section{Epidemiología}

Aunque la descripción inicial se atribuye a Pierre Marie en 1886, en dos casos propios más la recopilación de otros ocho previamente publicados en forma aislada, ${ }^{1}$ la acromegalia es una enfermedad que hasta

\footnotetext{
Jefe del Servicio de Endocrinología, Hospital de San José.
}

cierto punto ha sido protagonista de la historia de la humanidad. En la Biblia, la narración de David y Goliat puede ser el relato de la lucha entre una persona normal y un paciente acromegálico. Existen estudios sobre la posibilidad de que el faraón egipcio Akenathon y el emperador romano Maximinus Thrax, según las imágenes de monedas de la época, tuvieran acromegalia; la armada prusiana entre 1613 y 1640 reclutó a la población más alta de Europa para su ejército y en las pinturas de la misma son evidentes los rasgos acromegálicos de sus miembros. $^{2}$

Los estudios epidemiológicos sobre incidencia y prevalencia de la enfermedad son escasos, debido a que los pacientes consultan en forma tardía. En general, la incidencia anual está entre tres y cuatro casos nuevos por millón de habitantes ${ }^{3}$ y la prevalencia varía entre 40 y 90 casos por millón de habitantes. ${ }^{3,4}$

No existe diferencia en la presentación por sexo y por lo regular el tiempo promedio transcurrido entre la aparición de los cambios de la fisonomía y el diagnóstico es de ocho años. ${ }^{4}$

\section{Etiopatogenia}

La acromegalia es producida en el $98 \%$ de los casos por tumores hipofisiarios y en un mínimo porcentaje por causas extrahipofisiarias (Tabla 1).

\section{Patogénesis de los adenomas hipofisiarios}

En el individuo normal, la actividad hipofisiaria está regulada por las hormonas hipotalámicas, ya sean factores estimuladores como hormona liberadora de tirotropina (HLT), hormona liberadora de hormona del crecimiento (HLHC), hormona liberadora de gonadotropina (HLG) o inhibidores (dopamina). Aunque era de esperarse que ante una excesiva producción de los factores estimuladores se presentaran tumores hipofisiarios, la realidad había mostrado hasta hace poco su generación en pacientes con tumores ectópicos (próstata, pulmón, 
Tabla 1. Etiologia del exceso de hormona del crecimiento

\section{Intrahipofisiario (99\%)}

Hipersecreción de hormona del crecimiento

Adenoma denso (60\%)

Adenoma mixto ( $\mathrm{HC}$ y prolactina) $(25 \%)$

Adenoma mamosomatotropo (10\%)

Adenoma mixto plurihormonal (5\%)

Carcinoma hipofisiario productor de $\mathrm{HC}(<0,1 \%)$

Extrahipofisiario (1\%)

Hipersecreción de hormona del crecimiento

Adenoma hipofisiario ectópico

Producción ectópica por otros tumores (páncreas)

Aumento de hormona liberadora de hormona del crecimiento.

Eutópica (hamartomas hipotalámicos, gangliocitomas, gliomas)

Ectópica (tumor carcinoide, insulinoma, carcinoma de célula pequeña de pulmón)

\section{Pseudoacromegalia}

Exceso de actividad de factores de crecimiento

Resistencia a la insulina

Modificado de Drange M.R. Melmed S. Etiopatogenia. En Webb S. Acromegalia Editorial Acción Médica. Madrid 1998, Pág. 22

$\mathrm{HC}=$ Hormona del crecimiento de las características de estas células, se encuentran por lo regular alteraciones de los genes activadores o supresores de la proliferación celular que desencadenan la degeneración adenomatosa.

\section{Mutaciones en el gen que codifica} la proteína Gsp: la activación de la proteína G está involucrada en la activación de la adenilciclasa, enzima reguladora de la actividad de la HLHC sobre la síntesis y liberación de hormona de crecimiento (HC). Las mutaciones que produce esta alteración constitutiva, son cambios en el residuo 201 (arginina por histidina) o en el 227 (glicina por arginina o leucina). Este tipo de mutaciones es además una característica racial, pues en la población japonesa no se observa y en la caucásica es 10 veces más frecuente. Dicha mutación se encuentra en $40 \%$ de los tumores productores de $\mathrm{HC}$ y no se encuentra en prolactinomas, tumores productores de hormona estimulante del tiroides (HET) y es raro encontrarla en neoplasias no funcionantes. ${ }^{7,8}$

Proteínas de respuestaal AMPc activado: la síntesis de HC mediada por HLHC o por actividad de las proteínas Gsp, es regulada por los factores de trascripción nuclear en respuesta al AMPc (CREB). En estudios de adenomas hipofisiarios se ha encontrado aumento de la concentración de CREB activado, lo cual podría promover la transformación adenomatosa. ${ }^{7-10}$

\section{Anomalías del gen transformador de tumores} pituitarios (PTTG): este gen se encuentra expresado en forma anormal en todas las líneas celulares de tumores humanos, está ubicado en el cromosoma $5 q 33$ y constituye un indicador de malignidad. ${ }^{10}$

Otras alteraciones, como anormalidades del gen del retinoblastoma, de la proteína p53, y de los genes relacionados con esta proteína, los inhibidores de las quinasas dependientes de ciclinas y los factores de crecimiento fibroblástico, ${ }^{9}$ han sido estudiados y encontrados en otros adenomas hipofisiarios, pero su papel en la 
patogénesis de los tumores productores de $\mathrm{HC}$ no ha sido establecida. ${ }^{9}$

\section{Cuadro clínico}

El cuadro clínico de los pacientes con acromegalia se caracteriza por su aparición lenta, lo que hace que sólo cuando los cambios son muy notorios, acuda a la consulta. La mayoría de los estudios muestran que en promedio un paciente dura ocho años entre el momento en que aparecen los cambios fenotípicos hasta que se hace el diagnóstico. ${ }^{7,9,11}$

Las manifestaciones clínicas se pueden dividir en dos: aquellas producidas localmente por el efecto de masa hipofisiaria, y las sistémicas, causadas por el exceso de $\mathrm{HC}$ y del factor de crecimiento similar a la insulina (IGFI, por su sigla en inglés).

Los síntomas producidos por el efecto de masa son cefalea y alteraciones del campo visual, casi siempre como hemianopsia, debido a que los tumores son macroadenomas hipofisiarios que causan compresión del quiasma óptico. Aunque más raro, también se puede observar rinoliquia por erosión e invasión del tumor en el seno esfenoidal, diplopía o alteraciones de los pares craneanos, no por presión directa sino por compresión en los agujeros de la base del cráneo debido al crecimiento perióstico exagerado.

En cuanto a los síntomas causados por la hipersecreción de HC, podríamos decir que casi todos los órganos y sistemas están comprometidos en menor o mayor grado.

Sistema esquelético: es el que le da el nombre a la enfermedad por el crecimiento acral o de las partes distales. Así, habrá crecimiento de manos, pies (Figuras 1 y 2), de la mandíbula con prognatismo, diastema o separación de los dientes, mala oclusión dental y crecimiento de los huesos de la nariz (Figura 3). La proliferación de hueso cortical de la columna puede producir síndromes radiculares y la deformidad clásica del tórax en tonel es debida a la alteración de la columna torácica y la proliferación de los cartílagos costales. En las articulaciones se produce compromiso de una o varias con crepitación, dolor e hipermovilidad. Los derrames articulares son raros. Los estudios de patología muestran proliferación de condrocitos en el espacio articular con neoformación

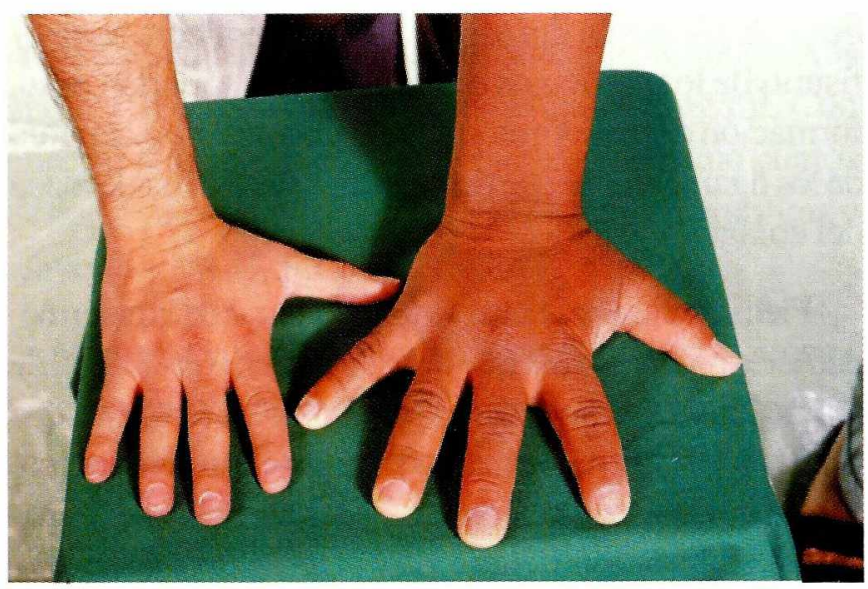

Figura I. Aumento de tamaño de las manos en un paciente acromegálico, comparado con una persona normal de la misma talla.

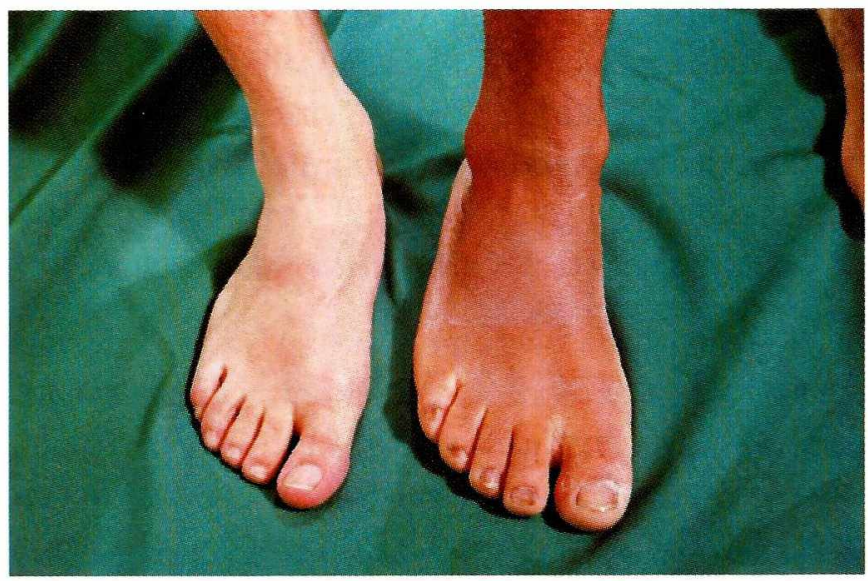

Figura 2. Aumento de tamaño de los pies en un paciente con acromegalia, comparado con persona normal de la misma talla.

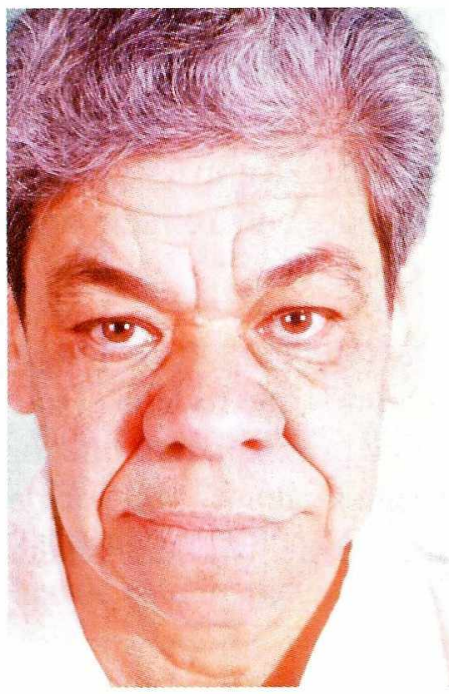

Figura 3. Fascies acromegálica. 
ósea, disminución del espacio articular, ulceraciones y fisuras de los cartílagos en las áreas que soportan peso, formación de osteofitos, quistes subcondrales y laxitud de los ligamentos peri articulares por síntesis inadecuada del colágeno. ${ }^{12,13}$

Piel: un síntoma frecuente y muy precoz de la enfermedad es la hiperhidrosis que se encuentra hasta en $88 \%$ de los pacientes, pero así mismo es uno de los síntomas que primero desaparecen con el tratamiento eficaz. Hay engrosamiento de la piel por depósito de mucopolisacáridos (ácido hialurónico, condroitinsulfato, glucosaminoglicanos), secreción exagerada de las glándulas sebáceas, aumento del tejido subcutáneo en el pie (almohadilla del talón o heel-pad) y engrosamiento de los dedos. ${ }^{12-14}$

Manifestaciones neuromusculares: como ya se mencionó, puede haber síndromes compartimentales del carpo o tarso, radiculopatías y la miopatía que se observa, con debilidad proximal, dolor y disminución de la tolerancia al ejercicio, es debida a atrofia de las fibras musculares tipo 2 e hipertrofia de las tipo 1. Puede haber estenosis del canal medular y síndrome de cauda equina. ${ }^{14-16}$

Aparato cardiovascular: el compromiso cardiovascular genera hasta el $60 \%$ de la morbimortalidad en los pacientes acromegálicos, y causa hipertensión arterial, cardiomegalia con hipertrofia miocárdica, fibrosis intersticial, hipertrofia septal asimétrica y disfunción diastólica. La enfermedad coronaria se encuentra en $20 \%$ de los enfermos con una mortalidad tres veces mayor que en la población general. ${ }^{13,16,17}$

Tracto respiratorio: las alteraciones son escasas, con engrosamiento y cambios estructurales en la mucosa y cartílagos de la laringe, que producen cambios en la voz haciéndola grave y gruesa. Las deformidades del tórax pueden producir una enfermedad pulmonar restrictiva.

Los cambios restantes son debidos a alteraciones de los tejidos blandos que rodean el tracto respiratorio superior, como prognatismo, macroglosia, hipertrofia de los cornetes y engrosamiento de las estructuras del cuello que pueden producir síndrome de apnea obstructiva del sueño. ${ }^{12,13}$

Tracto gastrointestinal: se encuentra colelitiasis hasta en $20 \%$ de los pacientes acromegálicos y es más frecuente que en la población general; por lo regular es asintomática. Es común el hallazgo de carcinoma del colon, pólipos hiperplásicos y adenomas túbulo-vellosos. Sin embargo, existe discusión sobre la verdadera incidencia de carcinoma de colon en estos pacientes, ya que hay estudios a favor y en contra de una mayor incidencia de esta neoplasia. ${ }^{18}$

Sistema reproductor: en las mujeres con acromegalia las alteraciones del ciclo menstrual tienen dos orígenes diferentes. Por una parte, los macroadenomas se asocian a déficit de gonadotropinas con o sin hiperprolactinemia, y por otro lado, hay una relación inversa entre los niveles de HC y los de proteína transportadora de esteroides sexuales. También se ha propuesto que algunas alteraciones son debidas al hiperinsulinismo que a menudo tienen estas pacientes. En el hombre, por los mismos mecanismos, habrá signos de hipogonadismo y disfunción eréctil..$^{19}$

Alteraciones metabólicas: al ser la HC una antagonista de la insulina, estos pacientes con frecuencia tienen alteración del metabolismo de los carbohidratos, que puede ir desde la intolerancia hasta la diabetes. Hay aumento de la síntesis de vitamina D3 activa, lo que incrementa la absorción de calcio en el intestino y produce hipercalciuria y urolitiasis. ${ }^{12,16}$

Para valorar el tratamiento, los cambios en algunos de los síntomas y signos son marcadores de respuesta adecuada, por lo que vale la pena mencionar cuales hallazgos pueden o no ser reversibles (Tabla 2).

\section{Diagnóstico}

Así como en la mayoría de los procesos diagnósticos en endocrinología, la presencia de un cuadro clínico característico, en este caso el paciente con fenotipo de acromegalia, nos obliga a confirmar la alteración hormonal con niveles elevados de HC. Para este fin, se puede hacer una medición aislada de la hormona en sangre periférica, pero debido a que la secreción de la HC es pulsátil, una determinación aislada no aporta información adecuada, por lo que hay que realizar pruebas específicas para confirmar el exceso de HC. ${ }^{11}$ Sin embargo, se deben tener en cuenta circunstancias en las cuales podríamos tener falsas mediciones positivas o negativas, las cuales están mencionadas en la Tabla 3. 
Tabla 2. Reversibitidad del cuadro clinico con tratamiento adecuado

\section{Artropatía}

Mejoría sintomática

Cambios irreversibles óseos y de cartilagos

\section{Cardiovascular}

Persisten cambios a pesar de niveles de $\mathrm{HC}$ normales

\section{Respiratorio}

Mejoría de síntomas. Enfermedad restrictiva persiste

\section{Neuropatía}

Mejoran los síndromes compartimentales

\section{Metabolismo}

Mejora intolerancia a los carbohidratos

Modificado de Melmed S. Acromegaly. En: DeGroot L, Jameson J. Endocrinology Editorial W.B.Saunders. Philadelphia, Fourth Ed, 200I, Pág. 304

Tabla 3. Casos en los que se encuentra elevada la HC sin acromegalla

\section{HC basal elevada}

Ingesta de estrógenos

Ingesta de L-Dopa

Hipoglucemia

Anorexia nerviosa

Malnutrición

Falla de supresión con la prueba de carga de glucosa

Insuficiencia renal crónica

Malnutrición

Anorexia nerviosa

Hepatopatía crónica

Respuesta elevada a la prueba de HLT

Adolescencia

Anorexia

Depresión

Porfiria intermitente aguda

Síndrome diencefálico

Modificado de Lucas T. Gigantismo y acromegalia. En: Jara Endocrinología, Editorial Médica Panamericana. Madrid, 2001, Pág. 56

Ya que normalmente durante una prueba de tolerancia oral a la glucosa, la $\mathrm{HC}$ disminuye a valores menores de $2 \mathrm{ng} / \mathrm{ml}$, este examen es útil para el diagnóstico, pues en la mayoría de los pacientes acromegálicos (80\%), los niveles nunca descenderán a menos de $2 \mathrm{ng} / \mathrm{ml}$. Con pruebas más sensibles, se han establecido como puntos de corte valores mayores de $0,057 \mathrm{ng} / \mathrm{ml}$ en hombres y mayores de $0,71 \mathrm{ng} / \mathrm{ml}$ en mujeres. ${ }^{11,16,20}$

Otra prueba útil es la medición del factor de crecimiento similar a la insulina tipo I ( IGF-I), conocido también como somatomedina $\mathrm{C}$, cuyos niveles en sangre periférica son más estables; como norma, niveles elevados en un paciente con sospecha clínica es casi siempre diagnóstico de acromegalia. Los valores de IGF-I deben compararse con cifras normales para la edad y el sexo. 12, 16,21,22

Una vez hecha la confirmación bioquímica de la hipersecreción de $\mathrm{HC}$, la presencia del adenoma se confirma mediante resonancia magnética simple y con contraste de la silla turca (Figura 4).

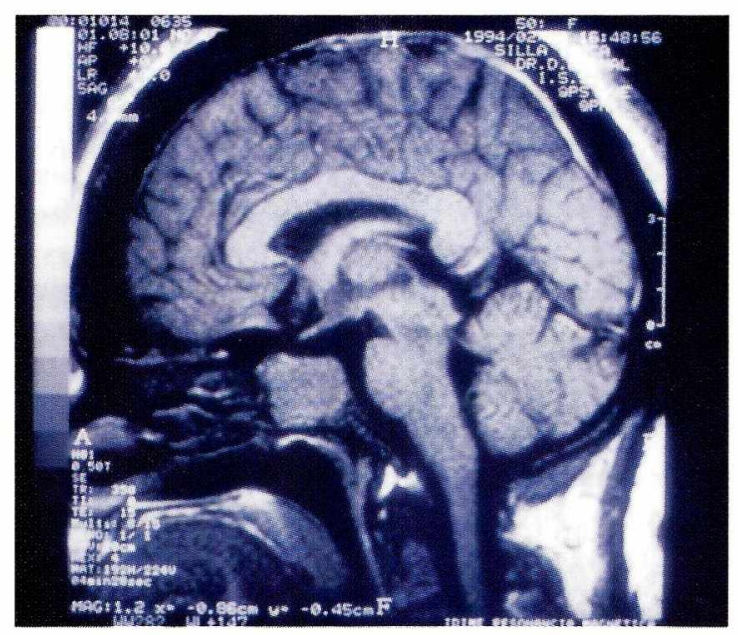

Figura 4. Resonancia nuclear magnética de un paciente con acromegalia. Se observa macroadenoma hipofisiario que invade el seno esfenoidal.

Como se mencionó en el cuadro clínico, el tiempo que transcurre entre el inicio de los síntomas y el diagnóstico, es aproximadamente de seis a ocho años, por lo tanto, es frecuente encontrar un macroadenoma hipofisiario. Cuando el tiempo de evolución es corto, es posible ver un microadenoma, o incluso puede encontrarse una silla turca normal, en los raros casos de producción ectópica de HLHC o HC. 


\section{Tratamiento}

El objetivo del tratamiento en la acromegalia está enfocado a manejar dos aspectos importantes: el efecto compresivo sobre las estructuras vecinas del adenoma y el control de la hipersecreción de HC.

Tratamiento quirúrgico: idealmente, el tratamiento para la acromegalia es la resección quirúrgica del adenoma con preservación de la función hipofisiaria. El abordaje quirúrgico más aceptado es la vía transesfenoidal. La mayoría de las series reportan haber logrado remisión de la enfermedad mediante tratamiento quirúrgico en $84 \%$ de los casos con microadenomas y en $64 \%$ de los macroadenomas, tomando como criterio de remisión los niveles de $\mathrm{HC}$ menores de $2 \mathrm{ng} / \mathrm{ml}$. ${ }^{23-25}$

Es importante aclarar que se menciona remisión de la enfermedad y no curación, debido a que durante el control postoperatorio con frecuencia se encuentran pacientes con supresión de la HC, pero con niveles aumentados de IGF-I. En los casos en que haya evidencia clínica de la enfermedad y persistencia de niveles altos de IGF-I, el paciente debe recibir 6 meses de tratamiento médico y una nueva evaluación.

El tratamiento no quirúrgico de la acromegalia se considera complementario a la cirugía y puede ser de dos tipos: farmacológico o con radioterapia.

Radioterapia externa: esta ha sido la compañera inseparable del tratamiento quirúrgico, en especial en los casos en que por dificultades técnicas o por las características del tumor, no se logra una resección completa. La dosis usual está entre 40 y 75 Gy y los resultados en cuanto a normalización de los niveles de HC y de IGF-I se observan en promedio a los ocho años; su efectividad se relaciona con los niveles de $\mathrm{HC}$ al momento de iniciar la radioterapia. ${ }^{26,29}$

Los principales riesgos de la radioterapia son el desarrollo de panhipopituitarismo, el compromiso o la pérdida visual y el daño cerebral. El panhipopituitarismo se puede observar en 50\% de los casos, sin que exista relación directa con la dosis recibida. La pérdida de la visión es del $2 \%$ y las anormalidades o daños cerebrales por la radiación alcanzan el $30 \%{ }^{28}$
Tratamiento médico: Existen en la actualidad tres tipos de medicamentos que pueden utilizarse en el manejo de la acromegalia: agonistas de la dopamina, análogos de la somatostatina y antagonistas del receptor de HC.

- Agonistas de la dopamina: dentro de este grupo están la bromocriptina, quinagolide, pergolide y cabergolide. Los mejores resultados terapéuticos se han obtenido con cabergolide. ${ }^{30-32}$

- Análogos de la somatostatina: la somatostatina es una hormona producida en las células de los islotes del páncreas y tiene como efecto principal bloquear la liberación de HLHC y de HC a nivel central. Gracias a esta característica, se han desarrollado compuestos análogos, que se unen con alta afinidad a los receptores de tipo SSR2 y SSR5. ${ }^{32,33}$ Dentro de estos se encuentran el octreótide, el lanreótide, el vapreótide y el octreótide de acción prolongada. El octreótide se aplica en forma subcutánea, por lo regular cada ocho horas. Su vida media es de seis a ocho horas, por lo que son necesarias tres dosis diarias para suprimir los niveles de HC. El lanreótide tiene una vida media más prolongada, de 14 a 21 días, motivo por el cual se puede aplicar una dosis intramuscular cada dos a tres semanas. El vapreótide se encuentra en fase III de investigación y tiene un efecto más prolongado, mientras que el octreótide de acción prolongada (Sandostatina LAR®) se puede aplicar siguiendo un esquema de una dosis cada cuatro semanas. ${ }^{33,34}$

La terapia con los análogos de la somatostatina ha sido utilizada como complementaria en casos en los que la resección quirúrgica no fue completa o en aquellos casos en que no se puede realizar resección parcial del tumor. Los últimos estudios ${ }^{33-35}$ han demostrado, incluso, que se puede utilizar este tipo de tratamiento como primera elección, debido a la respuesta rápida de los niveles de HC e IGF-I, con control hasta en $69,2 \%$ y $73,7 \%$, respectivamente ${ }^{35}$ y por los pocos efectos secundarios demostrados, los cuales son casi siempre gastrointestinales y están directamente relacionados con la dosis. Se puede observar diarrea, dolor abdominal y náuseas. La colelitiasis se puede considerar como la 
complicación más importante con el uso de octreótide y octreótide de depósito. Esto se debe a que la droga incrementa la saturación biliar de colesterol, favorece la formación de cristales y de cálculos, y retarda el vaciamiento de la vesícula al bloquear la actividad de la colecistoquinina, lo que favorece la litogénesis. ${ }^{34,35}$

- Antagonistas del receptor de HC: con el entendimiento de los mecanismos moleculares de acción de la HC sobre su receptor, se desarrollaron compuestos análogos de la HC con efecto completamente antagónico y con una alta afinidad por el receptor de HC. Una de estas moléculas es el pegvisomat, que se encuentra en fase de investigación clínica y ha logrado resultados significativos sobre los niveles de IGF-I en terapias de 12 semanas de duración, aunque incrementa los niveles de HC. En algunos pacientes se ha encontrado alteración de las pruebas de función hepática, por lo que su aplicación clínica todavía esta en estudio. ${ }^{36}$

Por último, se hace énfasis en que todas las medidas anteriores, ya sea cirugía, radioterapia complementaria o tratamiento médico, van encaminadas a curar al paciente que padece acromegalia, es decir, buscan disminuir los niveles de IGF-I y de HC a rangos normales, y acercar su morbimortalidad a los valores de la población general. Por lo tanto, se puede definir curación cuando los valores de IGF-I se encuentran en rangos normales ajustados para la edad y el sexo, y hay una supresión de los niveles de $\mathrm{HC}$ con cifras menores de $1 \mathrm{ng} / \mathrm{ml}$ durante una curva de tolerancia a la glucosa. ${ }^{37,38}$

\section{REFERENCIAS}

1. De Pablos P.L. Epidemiología. En: Webb S. Acromegalia. Madrid: Editorial Acción Médica; 1998. p. 9.

2. Sheaves R. A history of acromegaly. Pituitary 1999;2:7-28.

3. Alexander L, Appleton D, Halla R, Ross WM. Epidemiology of acromegaly in the New Castle region. Clin Endocrinol 1980;12:71-79.

4. Holdaway IM, Rajasoorya C. Epidemiology of acromegaly. Pituitary 1999;2:29-41.

5. Levy A. Monoclonality of endocrine tumours: what does it mean? Trends in Endocrinology and Metabolism 2001;12:301-7.

6. Frohman L, Kineman R. Growth hormone-releasing hormone and pituitary development, hyperplasia and tumorigenesis. Trends in Endocrinology and Metabolism 2002;13:299-303.
7. Shimon I, Melmed S. Genetic basis of endocrine disease. Pituitary tumor pathogenesis. J Clin Endocrinol Metab 1997;82:1675-81.

8. Hayward B, Barlier A, Korbonits M, Grossman A, Jacquet P, Enjalbert A, Bonthron D. Imprinting of the Gs" gene GNAS1 in the patogenesis of acromegaly. J Clin Invest 2001;107: R31-37.

9. Drange M, Melmed S. Molecular pathogenesis of acromegaly. Pituitary 1999,2: 43-50.

10. Drange M, Melmed S. Etiopatogenia. En: Webb S Acromegalia. Madrid: Editorial Acción Médica; 1998. p. 21-37.

11. Melmed S, Jackson I, Kleinberg D, Klibanski A. Current treatment guidelines for acromegaly. J Clin Endocrinol Metab 1998;83: 2646-52.

12. Ben-Shlomo A, Melmed S. Acromegaly. Endocrinol Metab Clin North AM 2001;30: 565-583.

13. Melmed S. Acromegaly. En: Melmed S: The pituitary. Cambridge: Editorial Blackwell Science; 1995. p.413-442.

14. Gómez LA, García H. Clínica. En: Webb S: Acromegalia. Madrid: Editorial Acción Médica; 1998. p. 55-83.

15. Lucas T. Gigantismo y acromegalia. En: Jara Endocrinología. Madrid: Editorial Médica Panamericana; 2001. p. 56.

16. Melmed S. Acromegaly. En: DeGroot L, Jameson J. Endocrinology. Fourth Edition. Philadelphia: Editorial W.B.Saunders; 2001.p. 300-312.

17. Colao AM, Marzullo P, Di Somma C, Lombardi G. Growth hormone and the heart. Clin Endocrinol 2001;54:137-54.

18. Renehan A, Bhaskar P, Painter J, O'Dwyer S, Haboubi N, Varma J, Ball S, Shalet $\mathrm{S}$. The prevalence and characteristics of colorectal neoplasia in acromegaly J Clin Endocrinol metab 2000; 83:3417-24.

19. Kaltsas G.A, Mukherjee P, Jenkins M, Satta A, Islam N, Monson J, Besser G, Grossman A. Menstrual irregularity in women with acromegaly. J Clin Endocrinol Metab 1999; 84:2731-5.

20. Chapman IM, Hartmann MI, Straue M, Johnson MI, Velhuis JD, Thorner MO. Enhanced sensitivity growth hormone (GH) chemiluminiscense assays reveals lower postglucose nadir GH concentrations in men than in women. J Clin Endocrinol Metab 1994; 78:1312-7.

21. Daughaday WH, Starkey RH, Saltman S, Gavin III JR, MillsDunlap B, Heath-Monnig E. Characterization of serum growth hormone and insuline-like growth factor I in active acromegaly with minimal elevation of serum GH J Clin Endocrinol Metab 1987; 65:617-23.

22. Patel Y, Ezzat S, Chik C, Rorstad O, Serri O, Ur E, Wilkins E. Guidelines for the diagnosis and treatment of acromegaly: A Canadian perpective. Clin Invest Med 2000; 23:172-87.

23. Shimon I, Cohen Z, Ram Z, Hadani M. Transsphenoidal surgery for acromegaly: endocrinological follow-up of 98 patients. Neurosurgery 2001;48:1239-45.

24. Biermasz N, Van Dulken H, Roelfsema F. Ten year followup results of transsphenoidal microsurgery in acromegaly. J Clin Endocrinol Metab 2000;85:4596-602. 
25. Ahmed S, Elsheikh M, Stratton M, Page RC, Adams CB, Wass JA. Outcome of transsphenoidal surgery for acromegaly and its relationship to surgical experience. Clin Endocrinol 1999; 50:561-7.

26. Epaminonda P, Porreti S, Capiello V, Beck-Peccoz P, Faglia $\mathrm{G}$, Arosio M. Efficacy of radiotherapy in normalizing serum IGF-I, acid-labile subunit (ALS) and IGFBP-3 levels in acromegaly. Clin Endocrinol 2001;55:183-9.

27. Peacey S, Toogood A, Veldhuis J, Thorner M, Shalet S. The relationship between 24 -hour growth hormone secretion and insulin-like growth factor $I$ in patients with successfully treated acromegaly: impact of surgery or radiotherapy. J Clin Endocrinol Metab 2001;86:259-66.

28. Jaffe C. Reevaluation of conventional pituitary irradiation in the therapy of acromegaly. Pituitary 1999; 2:55-62.

29. Biermasz N, Van Dulken H, Roelfsema F. Long term followup results of postoperative radiotherapy in 36 patients with acromegaly. J Clin Endocrinol Metab 2000;85:2476-82.

30. Colao A, Ferone D, Maezullo P, et al. Effect of different dopaminergic agents in the treatment of acromegaly. J Clin Endocrinol Metab 1997;82:518-23.

31. Abs R, Verhelst $\mathbf{J}$, Maiter $\mathrm{D}$ et al. Cabergoline in the treatment of acromegaly: a study in 64 patients. J Clin Endocrinol metab 1998;82:374-8.
32. Van der Lely A, De Herder W, Lamberts S. New medical treatment for acromegaly. Pituitary 1999;2:89-92.

33. Newman C. Medical therapy for acromegaly. Endocrine Metab Clin North Am 1999;28: 171-90.

34. Ayuk J, Stewart S, Stewart P, Sheppard M. Long-term safety and efficacy of depot long-acting somatostatin analogs for the treatment of acromegaly. J Clin Endocrinol Metab 2002;87:4142-6.

35. Colao A, Ferone D, Marzullo P, Cappabianca P, Cirillo S, Boerlin V, Lancranjan I, Lombardi G. Long term effects of depot long-acting somatostatin analog octreotide on hormone levels and tumor mass in acromegaly. J Clin Endocrinol Metab 2001; 86:2779-86.

36. Van der Lely A, Hutson K, Trainer P, Besser M, Barkan A, et al. Long term treatment of acromegaly with pegvisomat, a growth hormone receptor antagonist. Lancet 2001;358:1754-9.

37. Giustina A, Barkan A, Casanueva F, Cavagnini F, Frohman L, Ho K, Veldhuis J, Wass J, Von Werder K, Melmed S. Consensus. Criteria for cure of acromegaly: a consensus statement. J Clin Endocrinol Metab 2000;85:526-9.

38. Melmed S, Casanueva F, Cavagnini F, Chanson P, Frohman L, Grossman A, Ho K, Kleinberg D, Lamberts S, Laws E, Lombardi G, Vance LK, Von Werder K, Wass J, Guistina A. Consensus. Guidelines for acromegaly management. J Clin Endocrinol Metab 2002;87:4054-8. 